\title{
A Survey on Rainfall Prediction using Artificial Neural Network
}

\author{
Deepak Ranjan Nayak \\ College of Engineering and \\ Technology \\ Bhubaneswar, Odisha \\ India, 751003
}

\author{
Amitav Mahapatra \\ College of Engineering and \\ Technology \\ Bhubaneswar, Odisha \\ India, 751003
}

\author{
Pranati Mishra \\ College of Engineering and \\ Technology \\ Bhubaneswar, Odisha \\ India, 751003
}

\begin{abstract}
Rainfall prediction is one of the most important and challenging task in the modern world. In general, climate and rainfall are highly non-linear and complicated phenomena, which require advanced computer modeling and simulation for their accurate prediction. An Artificial Neural Network (ANN) can be used to predict the behavior of such nonlinear systems. ANN has been successfully used by most of the researchers in this field for the last twenty-five years. This paper provides a survey of available literature of some methodologies employed by different researchers to utilize ANN for rainfall prediction. The survey also reports that rainfall prediction using ANN technique is more suitable than traditional statistical and numerical methods.
\end{abstract}

\section{General Terms}

Rainfall, Artificial Neural Network, Prediction.

\section{Keywords}

Rainfall, Neural Network, BPN, RBF, SVM, SOM, ANN.

\section{INTRODUCTION}

Rainfall brings the most important role in the matter of human life in all kinds of weather happenings. The effect of rainfall for human civilization is very colossal. Rainfall is natural climatic phenomena whose prediction is challenging and demanding. Accurate information on rainfall is essential for the planning and management of water resources and also crucial for reservoir operation and flooding prevention. Additionally, rainfall has a strong influence on traffic, sewer systems and other human activities in the urban areas. Nevertheless, rainfall is one of the most complex and difficult elements of the hydrology cycle to understand and to model due to the complexity of the atmospheric processes that generate rainfall and the tremendous range of variation over a wide range of scales both in space and time. Thus, accurate rainfall prediction is one of the greatest challenges in operational hydrology, despite many advances in weather forecasting in recent decades. Rainfall means crops; and crop means life. Rainfall prediction is closely related to agriculture sector, which contributes significantly to the economy of the nation.

On a worldwide scale, large numbers of attempts have been made by different researchers to predict rainfall accurately using various techniques. But due to the nonlinear nature of rainfall, prediction accuracy obtained by these techniques is still below the satisfactory level. Artificial neural network algorithm becomes an attractive inductive approach in rainfall prediction owing to their highly nonlinearity, flexibility and data driven learning in building models without any prior knowledge about catchment behavior and flow processes. Artificial neural networks have been successfully used in these days in various aspects of science and engineering because of its ability to model both linear and non-linear systems without the need to make assumptions as are implicit in most traditional statistical approaches. ANN has been used as an effective model over the simple linear regression model. This paper provides a literature survey on rainfall prediction using different neural networks used by different researchers. The paper also discusses the concept of some neural network architectures briefly which will be helpful to the new researchers in this field. The objective of this survey is to make the prediction of rainfall more accurate in the recent future. The paper has been constructed with the sections. Section II discussed the concept of neural network. Different methodologies used by researchers to predict rainfall has been discussed in section III. Section IV discusses the literature survey of rainfall prediction all over the world. At last a conclusion is discussed in the section $\mathrm{V}$.

\section{CONCEPT OF ARTIFICIAL NEURAL NETWORK}

Soft computing deals with approximate models where an approximation answer or result is achieved. Soft computing has three basic components, namely, Artificial Neural Network (ANN), Fuzzy logic and Genetic Algorithm. ANN is commonly used by researchers in the field of rainfall prediction.

Human brain is a highly complex, nonlinear, and parallel computer (information-processing system). Neural Networks are simplified models of biological neuron system. A neural network is a massively parallel distributed processor made up of simple processing units, which has a natural propensity for storing experiential knowledge and making it available for use [1].The fundamental processing element of an ANN is an artificial neuron. Just like the natural neuron in human brain, it can receive inputs, process them and produce the relevant output. A simple mathematical model in Fig. 1 can be used in explaining a neuron quantitatively. The three basic elements of the neuronal model are: a set of synapses or connecting links, an adder and an activation function. This model also includes an externally applied bias, denoted by $b_{\mathrm{k}}$. 


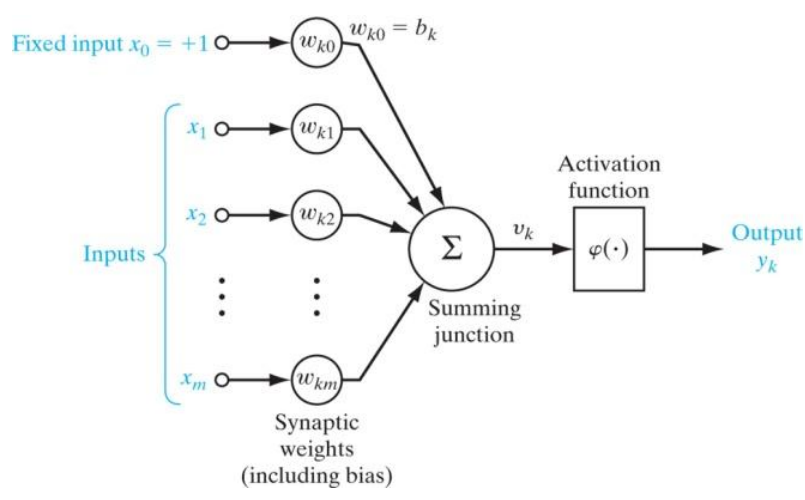

Fig 1: Nonlinear model of a neuron

The net input at the summing junction can be written as:

$$
u_{k}=\sum_{j=1}^{m} w_{k j} x_{j}
$$

and

$$
v_{k}=u_{k}+b_{k}
$$

The net input is then applied to an activation function, whose main objective is to limit the amplitude of the neuron to some finite value, and helps in achieving exact output. The output of the $\mathrm{k}^{\text {th }}$ neuron is:

$$
y_{k}=\varphi\left(u_{k}+b_{k}\right)
$$

A network of neurons is formed when a neuron links with others via synapses which can be single layered or multi layered. A multilayer ANN contains an input layer of neurons, an output layer of neurons, and one or more hidden layer of neurons. The hidden layer aids in performing useful intermediary computations before directing the input to the output layer.

When a network had been constructed for a specific application, inputs and the corresponding targets are used to train a network until it learns to associate a particular input with a reasonable output. A network is ideally trained until the change in weights in a training cycle reaches a minimum value. After the network is properly trained, it has to be tested for its ability to produce accurate outputs. Large multilayered networks with multiple nodes in each layer are capable of memorizing data due to the vast number of synaptic weights available on such a network. Thus, generating correct outputs for input vectors encountered within the training process does not justify the ability of a network to generate accurate outputs.

\section{METHODOLOGY}

The followings are the common types of neural networks used by different researchers for rainfall predictions.

\subsection{Back Propagation Network (BPN)}

The back-propagation learning algorithm is one of the most important developments in neural networks [2]. This network is still the most popular and most effective model for complex, multi layered networks. This learning algorithm is applied to multilayer feed-forward networks consisting of processing elements with continuous differentiable activation functions. The networks associated with back-propagation learning algorithm are also called back-propagation networks (BPNs). It is a supervised learning method. For a given set of training input-output pair, this algorithm provides a procedure for changing the weights in a BPN to classify the given input patterns correctly.

The basic concept of this algorithm is, it consists of two passes through the different layers of the network: a forward pass and a backward pass. In the forward pass, an input vector is applied to the sensory nodes of the network and its effect propagates through the network layer by layer. Finally a set of outputs produced as the actual response of the network. During the forward pass the synaptic weights of the networks are all fixed. During the backward pass, on the other hand, the synaptic weights are all adjusted in accordance with an error correction rule. Specifically, the actual response of the network is subtracted from the desired (target) response to produce an error signal. This error signal is then propagated backward through the network, against the direction of synaptic connections, hence the name "error backpropagation". The synaptic weights are adjusted to make the actual response of the network move closer to the desired response in a statistical sense [1].

The typical back-propagation network contains an input layer, an output layer, and at least one hidden layer. The number of neurons at each layer and the number of hidden layers determine the networks ability on producing accurate outputs for a particular data set. Most of the researchers have been used this network for rainfall prediction.

\subsection{Radial Basis Function Networks (RBFN)}

RBF Networks are the class of nonlinear layered feed forward networks. It is a different approach which views the design of neural network as a curve fitting problem in a high dimensional space. The hidden units provide a set of "functions" that constitute an arbitrary "basis" for the input patterns (vectors) when they are expanded to the hidden space, these functions are called radial-basis functions. The construction of a RBF network involves three layers with entirely different roles: the input layer, the only hidden layer, and the output layer ([3], [4]).

When a RBF network is used to perform a complex pattern classification task, the problem solved by transforming it into a high dimensional space in a nonlinear manner. RBF networks and MLPs (Multi Layer Perceptrons) are examples of nonlinear layered feed forward networks. They are both universal approximators. However, these two networks differ from each other. An RBF networks has a single hidden layer, whereas an MLP may have one or more hidden layers. The hidden layer of an RBF network is nonlinear and the output layer is linear, where as the hidden and output layers of an MLP are usually all nonlinear [1]. Several researchers have used this network for accurate rainfall prediction and got valuable results.

\subsection{Support Vector Machine (SVM)}

Support Vector Machine is one of the important category of multi layer feed forward network. Like multi layer perceptrons and radial basis function networks, support vector machines can be used for pattern classification and nonlinear 
regression. Support Vector Machines (SVMs) developed by Vapnik and his co-workers has been used for supervised learning due to - (i) Better generalization performance than other NN models (ii) Solution of SVM is unique, optimal and absent from local minima as it uses linearly constrained quadratic programming problem (iii) Applicability to nonvectorial data (Strings and Graphs) and (iv) Few parameters are required for tuning the learning $\mathrm{m} / \mathrm{c}$.

Kernel Methods are a set of algorithms from statistical learning which include the SVM for classification and regression, Kernel PCA, Kernel based clustering, feature selection, and dimensionality reduction etc [1]. SVM is found to be a significant technique to solve many classifications problem in the last couple of years. Very few researchers of this field used this technique for rainfall prediction and got satisfactory result

\subsection{Self Organizing Map (SOM)}

Self Organizing Map is a special class of artificial neural network. These networks are based on competitive learning. The output neurons of network compete among themselves to be activated or fired, with the result that only one output neurons is on at any time. This neuron is called winning neuron. The weight vector associate with winning neurons only updated in the scheme "winner takes all". Based on unsupervised learning, which means that no human intervention is needed during the learning and that little need to be known about the characteristics of input data. In SOM the neurons are organized in one or two dimensional lattice.

SOM are data visualization technique invented by Prof. Teuvo Kohonen that reduces the dimensions of data through selforganizing neural networks. The way SOM go about reducing dimensions is by producing a map of usually 1-D or 2-Ds, which plot the similarities of the data by grouping similar data items together. So, SOMs accomplish two things, they reduce dimensions \& display similarities ([1], [5], [6]).

\section{LITERATURE SURVEY}

Hu (1964) started the implementation of ANN in weather forecasting. He used an adaptive system called Adaline for pattern classification. This system, when trained on 200 winter sea level pressure and 24-hr pressure change patterns covering the area from $25 \mathrm{~N}$ to $65 \mathrm{~N}$ and $110 \mathrm{~W}$ to $170 \mathrm{~W}$, was able to make "rain"-"no rain" forecasts for the San Francisco Bay area on 100 independent cases that compared favorably with the official U.S. Weather Bureau forecasts for the same periods. After this research, he suggested that adaptive systems have the capability of making useful predictions or specifications of weather without complete understanding of the dynamics or complete measurement of the physical parameters involved [8].

In 1991 Cook and Wolfe presented a neural network to predict average air temperatures. They used back-propagation learning algorithm for this purpose and got satisfactory result [9].

An Artificial Neural Network model has developed by McCann (1992) to give 3-7 $\mathrm{hr}$ forecast of significant thunderstorms on the basis of surface based lifted index and surface moisture convergence. The two neural networks produced by them were combined operationally at National Severe Storms Forecast Center, Kansas City, Missouri to produce a single hourly product and was found to enhance the pattern recognition skill [10].

An important research work in applying ANN for rainfall forecasting was undertaken by French et al. (1992), which employed a neural network to forecast two-dimensional rainfall, $1 \mathrm{~h}$ in advance. Their ANN model used present rainfall data, generated by a mathematical rainfall simulation model, as an input data. That work was, however, limited in a number of aspects. For example, there was a trade-off between the interaction and the training time, which could not be easily balanced. The number of hidden layers and hidden nodes seemed insufficient, in comparison with the number of input and output nodes, to reserve the higher order relationship needed for adequately abstracting the process. Still, it has been considered as the first contribution to ANN's application and established a new trend in understanding and evaluating the roles of ANN in investigating complex geophysical processes [11].

Chen and Takagi (1993) have proposed a feature based neural network approach for rainfall prediction in the area of the open sea near Shikoku, Japan. A four-layer neural network was used to automatically learn the internal relationship between geostationary meteorological satellite GMS data and rainfall intensity distribution. They have used Back propagation learning algorithm for training and infrared and visible imagery of GMS image as the input data to the network [12].

In 1994 Zhang and Scofield presented an artificial neural network (ANN) technique for heavy convective rainfall estimation and cloud merger recognition from satellite data. They have developed an Artificial Neural network expert system for Satellite-derived Estimation of Rainfall (ANSER) in the NOAA/NESDIS Satellite Applications Laboratory and found that using artificial neural network group techniques, the following can be achieved: automatic recognition of cloud mergers, computation of rainfall amounts that will be ten times faster, and average errors of the rainfall estimates for the total precipitation event that will be reduced to less that 10 per cent [13].

Michaelides et al. (1995) compared the performance of ANN with multiple linear regressions in estimating missing rainfall data over Cyprus. They have proposed a technique that can be put forward in order to generate a sufficiently long time series of rainfall records for those locations for which the existing time series is either discontinued (forward extension) or where the archives have a relatively recent start (backward extension). The method uses artificial neural networks for the estimation of daily rainfall at particular observation sites in Cyprus (termed target stations) using as input daily rainfall observations from neighboring sites that had a sufficiently long and complete archive of data (termed control stations). In this way, the technique can be used to fill in missing data from the rainfall observation network but also for checking suspected data by using the records from surrounding stations. This technique of using neural networks is contrasted to the traditional multiple linear regression method. Here, the target station was considered as the dependent variable and the control stations as the independent variables [14].

Kalogirou et al. (1997) implemented ANN to reconstruct the rainfall over the time series in Cyprus. They used feed forward multilayer neural networks for the estimation of precipitation in selected rainfall collecting stations in Cyprus. 
Archived data collected for nine years and six control stations distributed around a target station used for training a suitable artificial neural network. Different neural network architectures and learning rates were tested, aiming at establishing a network that results in the best reconstruction of missing rainfall records. Then they chose multiple hidden layer neural network architecture for this purpose. This kind of architecture adopted for solving problems with similar requirements. The parameters used for the training of the network were collected at each control station. Those were the Julian day, height, distance between target and control stations, and the precipitation. The correlation coefficient obtained for the training data set was 0.933 . The verification of the network was done by using unknown data for the target station. This was done for a year, whose data were excluded from the training set. The correlation coefficient for the unknown case was 0.961 . The prediction error was confined to less than $17.1 \mathrm{~mm}$ of precipitation which is considered as acceptable [15]. In another research work Venkatesan et al. (1997) have employed an ANN to predict the all India summer monsoon rainfall with different meteorological parameters as model inputs. They used multilayered feed forward neural networks trained with the error-backpropagation (EBP) algorithm. Three network models that used, respectively, 2, 3 and 10 input parameters which are known to significantly influence the Indian summer monsoon rainfall (ISMR) was constructed and optimized. Then they compared their results obtained rigorously with the statistical models. The predictions of network models indicated that they can serve as a potent tool for ISMR prediction [16].

Lee et al. (1998) applied ANN in rainfall prediction by splitting the available data into homogenous subpopulations. They proposed a divide -and-conquer approach where the whole region is divided into four sub-areas and each is modeled with a different method. For two larger areas, they have used radial basis function (RBF) networks to perform rainfall prediction. The other two smaller sub-areas, they have used a simple linear regression model to predict the rainfall. Then they have made a comparison in between these two techniques and revealed that RBF networks produced good predictions while the linear models poor predictions. The authors believed that their method was suitable for emergency conditions as well as long term management of contaminated regions [17].

In 1999 Koizumi proposed an ANN model using radar, satellite and weather-station data together with numerical products generated by the Japan Meteorological Agency (JMA) Asian Spectral Model and the model was trained using 1 -year data. It was found that the ANN skills were better than the persistence forecast (after $3 \mathrm{~h}$ ), the linear regression forecasts, and the numerical model precipitation prediction. As the ANN model was trained with only 1 year data, the results were limited. The author strongly believed that the performance of the neural network would be improved when more training data became available. It is still unclear to what extent each predictor contributed to the forecast and to what extent recent observations might improve the forecast [18].

In 2000 Toth et al. compared short-term rainfall prediction models for real-time flood forecasting. They applied three time series models, auto-regressive moving average (ARMA), ANN and k-nearest-neighbors (KNN) method for forecasting storm rainfall occurring in the Sieve River basin, Italy, in the period 1992- 1996 with lead times varying from 1 to $6 \mathrm{~h}$. The result showed that the ANN performed the best in the improvement of the runoff forecasting accuracy when the predicted rainfall was used as inputs of the rainfall run-off model [19].

Luk et al. (2001) have developed and compared three types of ANN suitable for rainfall prediction i.e. multilayer feed forward neural network (MLFN), Elman partial recurrent neural network (Elman) and time delay neural network (TDNN) [21]. In the same year, Abraham et al. used four soft computing methods: ANN using Scaled Conjugate Gradient Algorithm (ANNSCGA), Evolving Fuzzy Neural Network (EfuNN), Adaptive Basis Function Neural Network (ABFNN) and General Regression Neural Network (GRNN) for predicting the rainfall time series. They have used a regression technique called Multivariate Adaptive Regression Splines (MARS) that uses a specific class of basis functions as predictors. In the study, monthly rainfall was used as input data for training model. The authors analyzed 87 years of rainfall data in Kerala, a state in the southern part of the Indian Peninsula. The empirical results showed that neurofuzzy systems were efficient in terms of having better performance time and lower error rates 5 compared to the pure neural network approach. Nevertheless, rainfall is one of the 20 most complex and difficult elements of the hydrology cycle to understand and to model due to the tremendous range of variation over a wide range of scales both in space and time ([11], [22]).

Wong et al. (2003) established a rainfall prediction model using soft computing technique that uses ANN and Fuzzy Logic. They have used SOM first to divide the data into subpopulation and hopefully reduce the complexity of the whole data space to something more homogeneous. After classification, they have used BPNNs to learn the generalization characteristics from the data within each cluster. They extracted fuzzy rules for each cluster. The fuzzy rule base is then used for rainfall prediction. They have compared this method with an established method, which uses radial basis function networks and orographic effect ([17]). Their results showed that the proposed method could provide similar results from the established method. However, the authors revealed that their method has the advantage of allowing analyst to understand and interact with the model using fuzzy rules [23].

In 2004 Chistodoulou et al. used an idea is to predict rainfall rate by using weather radar instead of rain-gauges measuring rainfall on the ground. The neural SOM and the statistical KNN classifier were implemented for the classification task using the radar data as input and the rain-gauge measurements as output. The rainfall rate on the ground was predicted based on the radar reflections with an average error rate of $23 \%$. Ultimately, they have observed that the prediction of rainfall rate based on weather radar measurements is possible [24].

In 2005 Lin et al. have developed a neural network with two hidden layers to forecast typhoon rainfall and it has been found that, the forecasting model can produce reasonable forecasts [25].

Guhathakurta (2006) developed a Artificial Neural Network model for Long-Range Monsoon Rainfall Prediction for the Districts and Sub-Division Kerala based on the area weighted value of all district forecast. Finally, he has found that the performance of the model was better than the statistical technique [26]. Somvanshi et al. (2006) have presented tools for modeling and predicting the behavioral pattern in rainfall phenomena based on past observations. They have introduced 
two fundamentally different approaches for designing a model, the statistical method based on autoregressive integrated moving average (ARIMA) and the emerging computationally powerful techniques based on ANN. In order to evaluate the prediction efficiency, they made use of 104 years of mean annual rainfall data from year 1901 to 2003 of Hyderabad region (India). The models were trained with 93 years of mean annual rainfall data. The ANN and the ARIMA approaches were applied to the data to derive the weights and the regression coefficients respectively. The performance of the model was evaluated by using remaining 10 years of data. Finally, the study reveals that ANN model could be used as an appropriate forecasting tool to predict the rainfall, which out performs the ARIMA model [27]. Box and Jenkins developed a forecasting technique, called ARIMA model which is still very popular among hydrologists [7]. Kumarasiri et al. (2006) utilized an innovative technique for rainfall forecasting using Artificial Neural Networks based on feed-forward backpropagation architecture. Focus was set upon making successful predictions from the available data, not on incorporating the physical aspects of the atmosphere or the actual process of rainfall occurrence. Both short term and long term forecasting was attempted for ground level data collected by the meteorological station in Colombo, Sri Lanka. They have developed three Neural Network models: a one-dayahead model for predicting the rainfall occurrence of the next day, which was able to make predictions with $74.25 \%$ accuracy, and two long term forecasting models for monthly and yearly rainfall depth predictions with $58.33 \%$ and $76.67 \%$ accuracies within a 5\% uncertainty level. The authors believed that each of these models was extended to make predictions several time steps into the future, where accuracies were found to be decreasing with the number of time steps. They have also studied and presented the success rates and rainfall trends within the monsoon seasons [28].

In 2007 Paras et al. proposed a weather forecasting model using Neural Network. They have been predicted the weather parameters like maximum temperature, minimum temperature and relative humidity using the features extracted over different periods as well as from the weather parameter timeseries itself. The approach applied there uses feed forward artificial neural networks (ANNs) with back propagation for supervised learning using the data recorded at a particular station. The trained ANN was used to predict the future weather conditions. The results were very encouraging and it was found that the feature based forecasting model can make predictions with high degree of accuracy. The model could be suitably adapted for making forecasts over larger geographical areas [29]. Chattopadhyay (2007) formulated a feed forward Artificial Neural Network model to predict the average summer-monsoon rainfall in India. In formulating the ANN based predictive model, three-layer network has constructed with sigmoid non-linearity. The monthly summer monsoon rainfall totals, tropical rainfall indices and sea surface temperature anomalies have considered as predictors while generating the input matrix for the ANN. The data pertaining to the years 1950-1995 have explored to develop the predictive model. Finally, he compared the prediction performance of neural net with persistence forecast and Multiple Linear Regression forecast and the supremacy of the ANN established over the other processes [30]. In the same year Kumar et al. have presented an Artificial Intelligence approach for regional rainfall forecasting for Orissa state, India on monthly and seasonal time scales. They have employed an Artificial Neural Networks (ANNs) methodology to handle the highly non-linear and complex behavior of the climatic variables for forecasting the rainfall. Genetic Optimizer (GO) was used by them to optimize the ANN architecture [31]. In another research Chattopadhyay et al. (2007) developed an ANN model step-by-step to predict the average rainfall over India during summer-monsoon by exploring the data available at the website (http://www.tropmet.res.in). To develop this model, the monsoon months (June-August) data of year y have used to predict the average monsoon rainfall of year $(y+1)$. They have used $75 \%$ of the available data as training set and remaining $25 \%$ as test set. The model was trained up to 50 epochs. The learning rate parameter was fixed at 0.4 and the momentum rate was chosen 0.9. Finally, they have compared the performance of the neural net model with conventional persistence forecast and found that the Neural Net, in the form of Multilayer Perceptron was adroit in the prediction of monsoon rainfall over India [32].

Chattopadhyay and Chattopadhyay (2008a) constructed an ANN model to predict monsoon rainfall in India depending on the rainfall series alone. They have developed nineteen neural network models with variable hidden layer size. Total rainfall amounts in the summer monsoon months of a given year used as input and the average summer monsoon rainfall of the following year used as the desired output to execute a supervised back propagation learning procedure. After a thorough training and test procedure, a neural network with eleven nodes in the hidden layer was found to be the most proficient in forecasting the average summer monsoon rainfall of a given year with the said predictors. Finally, they have compared the performance of the eleven hidden- nodes threelayered neural network with the performance of the asymptotic regression technique. Ultimately they have concluded that the eleven-hidden-nodes three-layered neural network has more efficacy than asymptotic regression in the present forecasting task [33]. Hung et al. (2008) employed an Artificial Neural Network model to forecast rainfall for Bangkok, Thailand with lead times of 1 to $6 \mathrm{~h}$. A real world case study was set up in Bangkok; 4 years of hourly data from 75 rain gauge stations in the area were used to develop the ANN model. Ultimately, they have applied the developed ANN model for real time rainfall forecasting and flood management [34].

In 2009 Xinia et al. have proposed a new model based on empirical mode decomposition (EMD) and the RBF neural network (RBFN) for rainfall prediction. After simulation they have concluded that the method had a high accuracy in denoising and prediction of the rainfall sequence [35]. Karmakar et al. (2009) have developed a three layer perception feed forward back propagation deterministic and probabilistic artificial neural network models to predict longrange monsoon rainfall over the subdivision EPMB. 61 years data for 1945-2006 have used, of which the first 51 years (1945-1995) of data were used for training the network and data for the period 1996-2006 were used independently for validation. However they have found that the performance of the model in probabilistic forecast was better evaluated over deterministic forecast [36]. In a Case Study on Jarahi Watershed, Solaimani (2009) has studied Rainfall-runoff Prediction Based on Artificial Neural Network and he concluded that Artificial Neural Network method is more appropriate and efficient to predict the river runoff than classical regression model [37].

In 2010 Baboo and Shareef used back propagation neural network for predicting the temperature based on the training set provided to the neural network. They have used 200 training data in this research for training the artificial neural 
network. The training data were created from the data available in data set. After training the system, they have tested it by predicting some unseen day's temperature and found accurate results. This approach was able to determine the non-linear relationship that exists between the historical data (temperature, wind speed, humidity, etc.,) supplied to the system during the training phase and on that basis, make a prediction of what the temperature would be in future [38]. Vamsidhar et al. (2010) proposed a back propagation neural network model for predicting the rainfall based on humidity, dew point and pressure in the country INDIA. The architecture of the neural network used for prediction was 3:7:1(input node: hidden node: output node). The rainfall data have taken from the period 1901-2000 and the data was taken from the website (www.tyndall.ac.uk). They have used twothird of the data for training and one-third for testing. The numbers of training and testing patterns were 250 training and 120 testing. Ultimately, they have obtained $99.79 \%$ of accuracy in the training and $94.28 \%$ of accuracy in testing. From these results the authors could able to predict the rainfall for the future [39]. Wu et al. (2010) have attempted attempt to seek a relatively optimal data-driven model for rainfall forecasting from three aspects: model inputs, modeling methods, and data preprocessing techniques. Four rain data records from different regions of India and China, namely two monthly and two daily series, were examined. They have made a comparison of seven input techniques and found that linear correlation analysis (LCA) was capable of identifying model inputs reasonably. A proposed model, modular artificial neural network (MANN), was compared with three models; artificial neural network (ANN), K-nearest-neighbors $(\mathrm{K}-\mathrm{NN})$, and linear regression (LR). Prediction was performed in the context of two modes including normal mode (without data preprocessing) and data preprocessing mode. From the normal mode they have got a result that MANN performs the best among all four models, but the advantage of MANN over ANN was not significant in monthly rainfall series forecasting. Under the data preprocessing mode, each of LR, $\mathrm{K}-\mathrm{NN}$ and ANN is respectively coupled with three data preprocessing techniques including moving average (MA), principal component analysis (PCA), and singular spectrum analysis (SSA). Results indicated that the improvement of model performance generated by SSA was considerable whereas those of MA or PCA were slight. Moreover, when MANN was coupled with SSA, results showed that advantages of MANN over other models were quite noticeable, particularly for daily rainfall forecasting [40]. To forecast rain intensity in Athens, Greece Panagiotis et al. (2010) have constructed predictive models using Artificial Neural Networks (ANNs) and they have proved that the results of the developed and applied ANN models showed a fairly reliable forecast of the rain intensity for the next four months [41]. Patil and Ghatol (2010) have used different artificial neural network topologies such as radial basis functions and multilayer perceptron with Levenberg Marquardt and momentum learning rules for rainfall forecasting using local parameters and found that the topologies fit for the same task [42].

Chadwick et al. (2011) employed an artificial neural network approach to downscale GCM temperature and rainfall fields to regional model scale over Europe [43]. A novel modular-type Support Vector Machine (SVM) have presented by Lu and Wang (2011) to forecast monthly rainfall in the Guangxi, China. Ultimately, they have showed that the prediction by using the SVM combination model was generally better than those obtained using other models presented in terms of the same evaluation measurements. The authors strongly believed that it could be used as an alternative forecasting tool for a Meteorological application in achieving greater forecasting accuracy and improving prediction quality further [44]. ElShafie et al. (2011) have developed two rainfall prediction models i.e. Artificial Neural Network model (ANN) and Multi Regression model (MLR) and implemented in Alexandria, Egypt. They have used statistical parameters such as the Root Mean Square Error, Mean Absolute Error, Coefficient Of Correlation and BIAS to make the comparison between the two models and found that the ANN model shows better performance than the MLR model [45]. A committee of artificial neural networks (ANNs) based model with wavelet decomposition was proposed by Charaniya et al. (2011) for prediction of monthly rainfall on accounts of the preceding events of rainfall data. Wavelet transform was used for extraction of approximate and detail coefficient of the rainfall data series. These coefficients were used along with a ANN for learning and knowledge extraction processes. They have tested the model on rainfall data for different geographical region of India and also for entire country and found that the proposed model was capable of forecasting monthly rainfall one month in advance [46]. El-Shafie et al. (2011) compared and studied two different static neural networks and one dynamic neural network namely; Multi-Layer Perceptron Neural network (MLP-NN), Radial Basis Function Neural Network (RBFNN) and Input Delay Neural Network (IDNN), respectively. Those models had been developed for two time horizon in monthly and weekly rainfall basis forecasting at Klang River, Malaysia. Ultimately, they concluded that IDNN could be suitable for modeling the temporal dimension of the rainfall pattern, thus, provides better forecasting accuracy [47]. Geetha and Selvaraj (2011) developed a back propagation neural network model for rainfall prediction in Chennai, India. The mean monthly rainfall was predicted by them using that model. The model can perform well both in training and independent periods [48]. El-shafie et al. (2011) have tried to use neural network and regression technique for rainfall-runoff prediction and finally they concluded that the feed forward back propagation ANN can describe the behavior of rainfall-runoff relation more accurately than the classical regression model [49]. In another research integrated artificial neural network-fuzzy logic-wavelet model is employed to predict Long term rainfall by Afshin et al. (2011). The results of the integrated model showed superior results when compared to the two year forecasts to predict the six-month and annual periods. As a result of the root mean squared error, predicting the two-year and annual periods is 6.22 and 7.11, respectively. However, the predicted six months shows 13.15 [50].

Abhishek et al. (2012) have developed an ANN model to forecast average monthly rainfall in the Udupi district of Karnataka, India. In Udupi, the months of April to November were identified as the rainfall season with May, June, July, August, and October as the main monsoon seasons. Thus they have explored the data of these 8 months from 1960 to 2010. Ultimately, three algorithms were tested in multi-layer architecture: Back Propagation Algorithm (BPA), Layer Recurrent Network (LRN) and Cascaded Back-Propagation (CBP) and the authors have found that BPA is the best algorithm out of the three tested [51]. A Multilayer Perceptron Neural Network was proposed by Deshpande (2012) as an intelligent tool for predicting Rainfall Time Series. The samples of rainfall have collected from the authorized Government Rainfall monitoring agency in Yavatmal, Maharashtra state, India. Multi-step ahead (1, 5, 10, 20) predictions of this Rainfall Data series have carried out using 
the proposed Multilayer Perceptron Neural Network. They have seen that the performance measures such as Mean square error, and Normalized mean square error on testing as well as training data set for short term prediction were optimal in comparison with other network such as Jordon Elmann Neural Network, SOFM (Self organized feature map), RNN (Recurrent neural network) [53].

Chau et al. (2013) have employed several soft computing approaches for rainfall prediction. They have considered two aspects to improve the accuracy of rainfall prediction: (1) carrying out a data-preprocessing procedure and (2) adopting a modular modeling method. The proposed preprocessing techniques included moving average (MA) and singular spectrum analysis (SSA). The modular models were composed of local support vectors regression (SVR) models or/and local artificial neural networks (ANN) models. The ANN was used to choose data- preprocessing method from MA and SSA. Finally, they have showed that the MA was superior to the SSA when they were coupled with the ANN [54]. Singh and Borah (2013) have used feed-forward backpropagation neural network algorithm for Indian Summer Monsoon Rainfall (ISMR) forecasting. Based on this algorithm, they have proposed the five neural network architectures designated as BP1, BP2, BP3, BP4, BP5 using three layers of neurons (one input layer, one hidden layer and one output layer). The data set was trained and tested separately for each of the neural network architecture (BP1BP5). The forecasted results obtained for the training and testing data are then compared with existing model. Results of the proposed method clearly exhibit superiority of their model over the considered existing model. The seasonal rainfall values over India for next 5 years have also been predicted [55].

\section{CONCLUSION}

This paper reports a detailed survey on rainfall predictions using different neural network architectures over twenty-five years. From the survey it has been found that most of the researchers used back propagation network for rainfall prediction and got significant results. The survey also gives a conclusion that the forecasting techniques that use MLP, BPN, RBFN, SOM and SVM are suitable to predict rainfall than other forecasting techniques such as statistical and numerical methods. However some limitation of those methods has been found. The extensive references in support of the different developments of ANN research provided with the paper should be of great help to ANN researchers to accurately predict rainfall in the future.

\section{ACKNOWLEDGEMENTS}

The authors wish to express sincere thanks to Professor Prashanta Kumar Patra, Head of the Department of Computer Science and Engineering of College of Engineering and Technology for his continuous inspiration towards this work.

\section{REFERENCES}

[1] S. Haykin, 1999: Neural Networks- A Comprehensive Foundation. Addison Wesley Longman.

[2] D. Rumelhart, G. Hinton and R. Williams, (1986) Learning representations by back propagating errors, Nature, 323, 533-536.

[3] M. J. D. Powell, (1987) Radial basis functions for multivariable interpolation: a review. In J.C. Mason, and M.G. Cox (Eds.), Algorithms for Approximation, pp. 143-167. Oxford: Clarendon Press.
[4] J Park, and I Sandberg, (1991) Universal approximation using radial basis function networks. Neural Computation, 3 (2), 246-257.

[5] T. Kohonen, (1990), The self organizing map. Proceedings of IEEE, 78(9), 1464-1478.

[6] W. Patterson Dan, (1996), Artificial Neural Networks: Theory and Applications, Prentice Hall.

[7] G.E.P. Box \& G.M. Jenkins, 1970. Time Series Analysis, Forecasting and Control, Holden-Day, CA, San Francisco.

[8] M.J.C., Hu, Application of ADALINE system to weather forecasting,Technical Report, Stanford Electron, 1964.

[9] D.F. Cook and M. L. Wolfe, "A back-propagation neural network to predict average air temperatures", AI Applications, 5, 40-46, 1991.

[10] D.W. McCann, "A Neural Network Short-Term Forecast of Significant Thunderstorms", Weather and Forecasting, 7, 525-534, doi: 10.1175/1520-0434, 1992.

[11] M. N. French, W. F. Krajewski, and R. R. Cuykendall: Rainfall forecasting in space and time using neural network, J. Hydrol., 137, 1-31, 1992.

[12] Tao Chen, M Takagi, "Rainfall prediction of geostationary meteorological satellite images using artificial neural network", IGARSS, 1993, vol.3, 1247 1249.

[13] M. Zhang, and A. R. Scofield, "Artificial Neural Network techniques for estimating rainfall and recognizing cloud merger from satellite data", International Journal of Remote Sensing, 16,3241-3262, 1994.

[14] S. C. Michaelides, C. C. Neocleous \& C. N. Schizas, "Artificial neural networks and multiple linear regressions in estimating missing rainfall data." In: Proceedings of the DSP95 International Conference on Digital Signal Processing, Limassol, Cyprus. pp 668673, 1995.

[15] S. A. Kalogirou, C. Neocleous, C. N. Constantinos, S. C. Michaelides \& C. N. Schizas, A time series reconstruction of precipitation records using artificial neural networks. In: Proceedings of EUFIT '97 Conference, 8-11 September, Aachen, Germany. pp 2409-2413, 1997.

[16] C. Venkatesan, S.D. Raskar, S.S. Tambe, B.D. Kulkarni, R.N. Keshavamurty, 1997, Prediction of all India summer monsoon rainfall using error-back-propagation neural networks. Meteorol. Atmos. Phys. 62 (3-4), 225 240 .

[17] S. Lee, S. Cho \& P.M. Wong,"Rainfall prediction using artificial neural network", J. Geog. Inf. Decision Anal, 2, 233-242, 1998.

[18] K. Koizumi: “An objective method to modify numerical model forecasts with newly given weather data using an artificial neural network", Weather Forecast., 14, 109$118,1999$.

[19] E. Toth, A. Brath, A. Montanari," Comparison of shortterm rainfall prediction models for real time flood forecasting", Journal of Hydrology 239 (2000) 132-147. 
[20] K Gwangseob and P.B. Ana, "Quantitative flood forecasting using multisensor data and neural networks", Journal of Hydrology, 246, 45-52, 2001.

[21] Kin C Luk, J. E. Ball and A. Sharma, 2001, "An Application of Artificial Neural Networks for Rainfall Forecasting", 33, pp. 883-699.

[22] Ajith Abraham, Dan Steinberg and Ninan Sajeeth Philip,"Rainfall Forecasting Using Soft Computing Models and Multivariate Adaptive Regression Splines", 2001.

[23] K.W. Wong, P.M. Wong, T. D. Gedeon \& C. C. Fung, "Rainfall Prediction Using Soft Computing Technique", Soft Computing 7 (2003) $434-438$.

[24] C.I. Christodoulou, S.C. Michaelides, M. Gabella, C.S. Pattichis, "Prediction of rainfall rate based on weather radar measurements", IEEE, 2004, vol. 2, 1393-1396.

[25] Gwo-Fong Lin and Lu-Hsien Chen, 2005, "Application of an artificial neural network to typhoon rainfall forecasting”, Hydrological Processes, 19, pp. 1825-1837.

[26] P. Guhathakurta, 2006, "Long-Range Monsoon Rainfall Prediction of 2005 for the Districts and Sub-Division Kerala with Artificial Neural Network", Current Science, 90 (6), pp-773-779.

[27] V.K.Somvanshi, O.P.Pandey, P.K.Agrawal, N.V.Kalanker1, M.Ravi Prakash and Ramesh Chand, "Modeling and prediction of rainfall using artificial neural network and ARIMA techniques", J. Ind. Geophys. Union (April 2006) Vol.10, No.2, pp.141-151.

[28] A.D. Kumarasiri and D.U.J. Sonnadara, 2006, "Rainfall Forecasting: An Artificial Neural Network Approach", Proceedings of the Technical Sessions, vol 22, pp. 1-13.

[29] Paras, Sanjay Mathur, Avinash Kumar, and Mahesh Chandra, "A feature based on weather prediction using ANN"World Academy of Science, Engineering and Technology 342007.

[30] S Chattopadhyay, "Feed Forward Artificial Neural Network model to predict the average summer moonsoon rainfall in India”, Acta Geophysica 55 (3), 369-382.

[31] D.N. Kumar, M.J. Reddy, R. Maity, “ Regional Rainfall Forecasting using Large Scale Climate Teleconnections and Artificial Intelligence Techniques", Journal of Intelligent System, Vol. 16, No. 4, 2007.

[32] S Chattopadhyay and Manojit Chattopadhyay, "A Soft Computing technique in rainfall forecasting", International Conference on IT, HIT, March19-21, 2007.

[33] S. Chattopadhyay, G. Chattopadhyay, 2008a. Identification of the best hidden layer size for threelayered neural net in predicting monsoon rainfall in India. J. Hydroinformatics 10 (2), 181-188.

[34] N. Q. Hung, M. S. Babel, S. Weesakul, and N. K. Tripathi, 2008, "An artificial neural network model for rainfall forecasting in Bangkok, Thailand", Hydrology and Earth System Sciences, vol. 5, pp. 183-218.

[35] Liu Xinia, Zhang Anbing, Shi Cuimei, Wang Haifeng, "Filtering and Multi-Scale RBF Prediction Model of Rainfall Based on EMD Method", ICISE, 2009, 3785 3788 .
[36] S. Karmakar, M.K. Kowar, P. Guhathakurta, "LongRange Monsoon Rainfall Pattern Recognition and Prediction for the Subdivision 'EPMB' Chhattisgarh Using Deterministic and Probabilistic Neural Network “, ICAPR, 2009, 367-370.

[37] Karim Solaimani, 2009, "Rainfall-runoff Prediction Based on Artificial Neural Network (A Case Study: Jarahi Watershed)", American-Eurasian J. Agric. \& Environ. Sci., vol. 5(6), pp. 856-865.

[38] Dr S. Santosh Baboo and I. Khadar Shareef, "An efficient Weather Forecasting Model using Artificial Neural Network", International Journal of Environmental Science and Development, Vol. 1, No. 4, October 2010.

[39] Enireddy. Vamsidhar, K.V.S.R.P. Varma , P Sankara Rao, Ravikanth satapati, 2010, "Prediction of Rainfall Using Back propagation Neural Network Model", International Journal on Computer Science and Engineering, Vol. 02, No. 04, pp. 1119-1121.

[40] C. L. Wu, K. W Chau, and C. Fan, 2010, "Prediction of Rainfall Time Series Using Modular Artificial Neural Networks Coupled with Data Preprocessing Techniques", Journal of Hydrology, Vol. 389, No. 1-2, pp. 146-167.

[41] Nastos Panagiotis, Moustris Kostas, Larissi Ioanna, and Paliatsos Athanasios, 2010, "Rain intensity forecast using Artificial Neural Networks in Athens, Greece", Geophysical Research Abstracts, Vol. 12.

[42] C.Y. Patil and A.A Ghatol., 2010, "Rainfall forecasting using local parameters over a meteorological station: an artificial neural network approach", International J. of Engg. Research \& Indoor. Appls, Vol.3, No. II, pp 341356.

[43] R. Chadwick, E. Coppola, F. Giorgi, 2011. An artificial neural network technique for downscaling GCM outputs to RCM spatial scale. Nonlinear Processes Geophys. 18 (6), 1013-1028.

[44] Kesheng Lu, Lingzhi Wang, "A Novel Nonlinear Combination Model Based on Support Vector Machine for Rainfall Prediction”, CSO, 2011, 1343 - 1346.

[45] Amr H. El-Shafie, A. El-Shafie, Hasan G. El Mazoghi, A. Shehata and Mohd. R. Taha, 2011, "Artificial neural network technique for rainfall forecasting applied to Alexandria, Egypt", International Journal of the Physical Sciences Vol. 6(6), pp. 1306-1316, 2011.

[46] N.A. Charaniya, S.V. Dudul, "Committee of artificial neural networks for monthly rainfall prediction using wavelet transform”, ICBEIA, 2011, 125-129.

[47] A El-Shafie, A. Noureldin, M. R. Taha, and A. Hussain, 2011, "Dynamic versus static neural network model for rainfall forecasting at Klang River Basin, Malaysia", Hydrol. Earth Syst. Sci., vol. 8, pp. 6489-6532.

[48] G. Geetha,. R Samuel Selvaraj, 2011, "Prediction of monthly rainfall in Chennai using back propagation neural network model", International Journal of Engineering Science and Technology, Vol. 3 No. 1, pp. 211-213 
[49] A. El-shafie, M. Mukhlisin, A. Najah Ali and M. R Taha, 2011, "Performance of artificial neural network and

[50] regression techniques for rainfall-runoff prediction", International Journal of the Physical Sciences, Vol. 6(8), pp. 1997-2003.

[51] Afshin Sarah, Fahmi Hedayat, Alizadeh Amin, Sedghi Hussein and Kaveh Fereidoon, 2011, "Long term rainfall forecasting by integrated artificial neural network-fuzzy logic-wavelet model in Karoon basin", Scientific Research and Essays, Vol. 6(6), pp. 1200-1208.

[52] Kumar Abhishek, Abhay Kumar, Rajeev Ranjan, Sarthak Kumar, "A Rainfall Prediction Model using Artificial Neural Network", 2012 IEEE Control and System Graduate Research Colloquium.
[53] G.Shrivastava, S. Karmakar, M.K. Kowar, P. Guhathakurta, "Application of Artificial Neural Networks in Weather Forecasting: A Comprehensive Literature Review", IJCA, Vol 51,No. 18,09758887,2012 .

[54] Rohit R. Deshpande, "On the Rainfall Time Series Prediction Using Multilayer Perceptron Artificial Neural Network", IJETAE, vol. 2, 1, 2012

[55] C.L. Wu, K.W. Chau*, "Prediction of rainfall time series using modular soft computing methods", Engineering Application of Artificial Intelligence 26 (2013), 9971007.

[56] P Singh and B Borah, "Indian summer monsoon rainfall prediction using artificial neural network", Stochastic Environmental Research and Risk Assessment, 2013, 1436-3240. 